\title{
Polycarboxylate Based Superplasticizers as Dispersant Agents for Exfoliated Graphene Nanoplatelets Reinforcing Cement Based Materials
}

\author{
Z. S. Metaxa* \\ Laboratory of Structural Mechanics, School of Rural and Surveying Engineering, National Technical University of Athens, Zografou \\ Campus, 9 Heroon Polytechniou St, 15780 Athens, Greece
}

Received 6 December 2015; Accepted 30 December 2015

\begin{abstract}
Graphene nanoplatelets (GNPs) are considered one of the most advanced nanomaterials that hold the promise of providing multifunctional characteristics to the cementitious matrix. To effectively employ the GNPs as a nanoreinforcement, their uniform dispersion within the matrix must be achieved. The present study investigates the efficiency of four different polycarboxylate based superplasticizers, which are fully compatible with cement-based materials, to be exploited as GNPs dispersant agents. Exfoliated GNPs were selected that had a quite small diameter/lateral size of $\sim 5 \mu \mathrm{m}$. The dispersing efficiency of the superplasticizers was investigated experimentally by measuring the electrical resistivity of the resulting nanocomposites. A discussion explaining the dispersing mechanism of these types of surfactants is provided. The use of a superplasticizer in conjunction with ultrasonic energy application was found to be necessary to properly disperse the GNPs. The results prove that the polycarboxylate based superplasticizers can be employed to promote the GNPs uniform distribution in cementitious materials. The polycarboxylate ester superplasticizer showed poor results, while the polycarboxylate polymer superplasticizers were found to be more effective to uniformly disperse the GNPs.
\end{abstract}

Keywords: cement-based nanocomposites, exfoliated graphene nanoplatelets, electrical resistivity polycarboxylate-based superplasticizers

\section{Introduction}

Graphene is one of the most recent cutting-edge, innovative nanomaterials that can be used as reinforcement to develop multifunctional nanocomposites with improved mechanical and electrical properties. This is possible due to its exceptional stiffness (Young's modulus of $1 \mathrm{TPa}$ ), high ultimate strength $(130 \mathrm{GPa})$, high electrical conductivity $(6000 \mathrm{~S} / \mathrm{cm})$ and large specific surface area [1-4]. Moreover, the electrical properties of the graphene sheets are independent of their chirality making them suitable for the development of high-performance sensing materials [4].

A single graphene sheet is a two dimensional material with the thickness of one-atom consisting of $\mathrm{sp}^{2}$-hybridized carbon atoms forming a hexagonal lattice. Graphene is typically employed in the form of graphene nanoplatelets (GNPs) where few layers of single graphene sheets are stack together. The carbon atoms are strongly bonded in the hexagonal plane but weakly bonded normal to the plane [5]. The graphene nanoplatelets compared to other types of carbon fillers, such as carbon nanotubes (CNTs), are expected to have several distinct advantages as a reinforcing material for cementitious materials. They have greater mechanical properties than CNTs, which should improve the

\footnotetext{
* E-mail address: zmetaxa@gmail.com ISSN: 1791-2377 @ 2015 Kavala Institute of Technology. All rights reserved.
}

overall mechanical behaviour of the composite. Additionally, they can be produced at large quantities having high purity at a lower cost compared to CNTs.

A very important factor when using carbon based nanomaterials as reinforcement in composites is their dispersion state. Due to the very strong van der Waals interactions the as-produced GNPs have the tendency to selfassociate into agglomerates and bundles. To successfully produce innovative nanocomposites, disentanglement and uniform dispersion of the GNPs inside the matrix must be achieved. One main approach, that has been adopted by many researchers [6-11], to uniformly disperse CNTs in a cementitious matrix is based on the use of a surfactant, i.e. a superplasticizer (SP) compatible with the cement based matrix, and the application of ultrasonic energy. One objective of this study is to investigate whether a similar method can be applied to uniform disperse the graphene nanoplatelets.

Research on the potential of using the GNPs as reinforcement in cementitious matrices is still at its initial stages. Zohhadi et al. [12] were the first to report on the use of graphene nanoplatelets as a reinforcement in cement based composites. To minimize its hydrophobicity and facilitate dispersion in water, the GNPs were functionalized using ammonium persulfate, $\left(\mathrm{NH}_{4}\right)_{2} \mathrm{~S}_{2} \mathrm{O}_{8}$ and sulfuric acid, $\mathrm{H}_{2} \mathrm{SO}_{4}$. It was concluded that the number of graphene monolayers was significantly reduced, thus aiding in the dispersion process of GNPs in the cementitious matrix. Functionalization with nitric acid $\left(\mathrm{HNO}_{3}\right)$ was also adopted 
by Alkhateb et al. [13] for the dispersion of GNPs. Acid treatment, however, is known to impose defects on the graphene nanoplatelets, furthermore, the compatibility of these functionalized nanoplatelets having a low $\mathrm{pH}$ level inside a strong alkaline environment is not straightforward $[12,14]$. More recently, Le et al. [15] as well as Du and Pang [16], employed a naphthalene sulfonate-based superplasticizer (Darex Super 20) and ultra-sonication for exfoliation and dispersion of the GNPs. To ensure that all the mixes had similar flowability, Du and Pang [16] kept the amount of superplasticizer at $50 \%$ of the weight of GNPs. The GNPs were mixed in the mixing water containing the water reducer admixture and ultra-sonicated for one hour. According to visual inspection and analysis of the GNPs particle size distribution with and without ultra-sonication, the application of sonication and the use of a surfactant is necessary for their disentanglement.

Despite the research efforts to date, no attempts have been made to compare the dispersing power of different superplasticizers as GNPs dispersants for use in cementitious materials. Moreover, the superplasticizer that has been used so far for the GNPs dispersion in cement based materials (naphthalene sulfonate-based superplasticizer) operates according to the mechanism of electrostatic repulsion. This mechanism is known to be less effective compared to other water reducing agents that disperse the cement particles through steric hindrance, i.e. the polycarboxylate based superplasticizers. Previous research has shown that a naphthalene based superplasticizer is not as effective in dispersing multi-walled CNTs compared to four different polycarboxylate based superplasticizers [9].

The effect of superplasticizer type as dispersing agent for graphene nanoplatelets that were produced through exfoliation was experimentally investigated in this study. GNPs with extra small diameter of $\sim 5 \mu \mathrm{m}$ were chosen in the present work because their dispersion is known to be more difficult [5]. Four different types of commercially available superplasticizers were examined. Electrical resistance measurements were conducted on the produced nanocomposites to study the dispersing efficiency of the different superplasticizer types.

\section{Experimental Procedure}

\subsection{Materials}

The GNPs/cement nanocomposites were prepared using Type I ordinary Portland cement and Grade $M$ graphene nanoplatelets supplied by XG sciences Inc, Michigan. The GNPs were produced with the exfoliation method and therefore in the following will be referred as exfoliated graphene nanoplatelets (xGnPs) as well. They had, according to the manufacturers' data sheet, an average thickness of approximately 6-8 nm, a typical surface area of 120 to $150 \mathrm{~m}^{2} / \mathrm{g}$ and average particle diameter of $5 \mu \mathrm{m}$. To facilitate with the $\mathrm{xGnPs}$ dispersion in an aqueous suspension, four different polycarboxylate based high range water reducer admixtures were used. The superplasticizers were kindly provided by Sika Hellas, having the following brand names: (i) ViscoCrete ${ }^{\circledR} 20 \mathrm{HE} \mathrm{S}$, (ii) ViscoCrete ${ }^{\circledR}$ Ferro 1000, (iii) ViscoCrete ${ }^{\circledR}$ Ultra 600 and (iv) ViscoCrete ${ }^{\circledR} 5500$ HP. The first one (ViscoCrete ${ }^{\circledR} 20$ HE S) is based on polycarboxylate ester the rest on polycarboxylate polymers (ViscoCrete ${ }^{\circledR}$ Ferro 1000 , ViscoCrete ${ }^{\circledR}$ Ultra 600 and ViscoCrete $\left.{ }^{\circledR} 5500 \mathrm{HP}\right)$.

\subsection{Preparation of the cementitious nanocomposites}

Before mixing with cement, the xGnPs were dispersed in an aqueous solution that contained the dispersant. To ensure that the volume of the suspension was limited, part of the mixing water, corresponding to $6 \%$ of the mixing water, was used in the solution. To homogeneous disperse the $\mathrm{xGnPs}$ in the above suspension, ultrasonic energy was applied using a probe ultrasonicator. The homogenizer was operating at $50 \%$ of its power, at cycles of $0.5 \mathrm{~s}$ to prevent heating of the suspensions. The same superplasticizer and $x \mathrm{GnPs}$ concentration was used in all the suspensions that was 0.6 and 0.1 by weight percentage of cement, respectively. Fig. 1 shows two typical $\mathrm{xGnP}$ suspensions with or without surfactant photographed 1 min after mixing before the application of ultrasonic energy. Without the use of superplasticizer (Fig.1a), due to their hydrophobic nature, the xGnPs are observed mainly at the top of the suspension. When a superplasticizer was used, the xGnPs immediately emerged in the aqueous solution indicating the good affinity between the xGnPs and the superplasticizer, Fig.1b. In both cases a number of $x \mathrm{GnPs}$ was sentimented at the bottom of the solution demonstrating that the application of ultrasonic energy is required for the disentanglement of the xGnPs. After sonication, the suspension with the superplasticizer was uniform and no xGnP agglomerates were observed.

After dispersion, extra water was added to the suspensions that corresponded to a water to cement ratio of 0.3 . The resulting mixture was hand stirred and mixed with the cement using a standard mixer according to ASTM C305. Following, the cement paste nanocomposites were placed in prismatic $20 \times 20 \times 80 \mathrm{~mm}$ molds. The specimens were demolded after one day and were placed in water saturated with lime until the age of 28 days. Prior to testing, the samples were placed in an electrical heating oven with air circulation at a temperature of $80 \pm 1^{\circ} \mathrm{C}$ to dry for 72 hours. At least three specimens were prepared for each case.

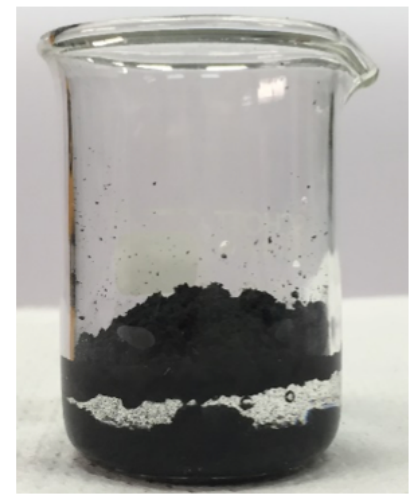

(a)

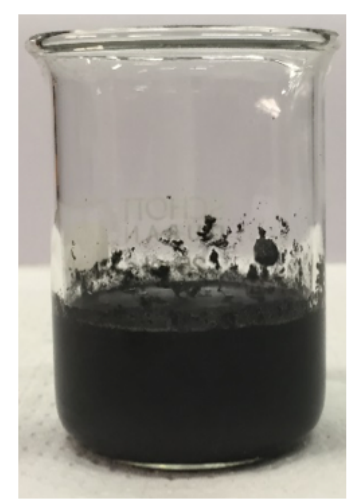

(b)
Fig. 1. Typical xGnP suspensions 1 min after mixing before the application of ultrasonic energy (a) without superplasticizer and (b) with superplasticizer.

\subsection{Electrical properties measurements}

The electrical resistance of the nanocomposites was measured using the four wire method. During casting, four steel electrodes were embedded into the samples covering the entire cross-section of the specimens. The distance between the outer and inner electrodes was $15 \mathrm{~mm}$ while the respective distance between the inner electrodes was $30 \mathrm{~mm}$, Figure 2. A rubber mat was placed at the bottom of the specimen in order to insulate the specimen. A Keysight multimeter was used for the four wire ohm measurements; the inner electrodes were used to measure the voltage and 
the outer electrodes to supply the current. The electrical resistance was recorded every second for a period of 30 minutes to avoid any discrepancies caused due to electric polarization effects. The resistivity $\rho$ of the nanocomposites was calculated by exploiting Ohm's law as $\rho=R \cdot S / L$, where $R$ is the electrical resistance, $S$ is the cross-section of the specimen and $L$ is the distance between the inner electrodes. To calculate the average resistivity values the data of the last five minutes of the measurements were used, as can be seen schematically in Fig. 3.

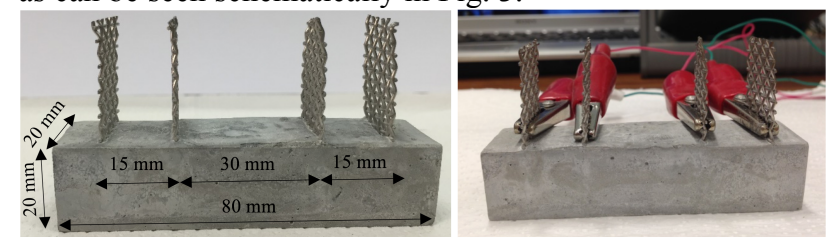

Fig. 2. (a) Specimen dimensions and (b) four point wire method for electrical resistance measurements.

\section{Results and Discussion}

The typical curves of the electrical resistivity over testing time for the plain cement paste, the nanocomposites dispersed only with the application of ultrasonic energy without using a superplasticizer and the nanocomposites dispersed with the different superplasticizers are shown in Fig 3. For the case of plain cement paste, high electrical resistivity values were recorded of the order of 1.5 $\mathrm{MOhm} \times \mathrm{cm}$. At the beginning of the test, higher values can be noticed of around $1.65 \mathrm{MOhm} \times \mathrm{cm}$. This can be attributed to the electrical polarization effect. Electric polarization refers to the phenomenon in which the centers of positive and negative charges do not coincide [17]. It typically occurs when a dielectric material is exposed to a direct current (DC) electric field. Consequently, the resistance of the material, especially at the beginning of the measurement is constantly changing. This polarization effect was also strongly evident for the sample without superplasticizer (grey curve) as well as for the $5500 \mathrm{HP}$ and the $20 \mathrm{HE} \mathrm{S}$ superplasticizers (blue and red curves, respectively). The resistivity is quite stabilized after few minutes during testing; however some scatter in the measurements can be noticed, especially for the sample without superplasticizer. The nanocomposites produced with Ferro 1000 and Ultra 600 superplasticizers demonstrate less scatter and lower electrical resistivity values as well as no polarization effect.

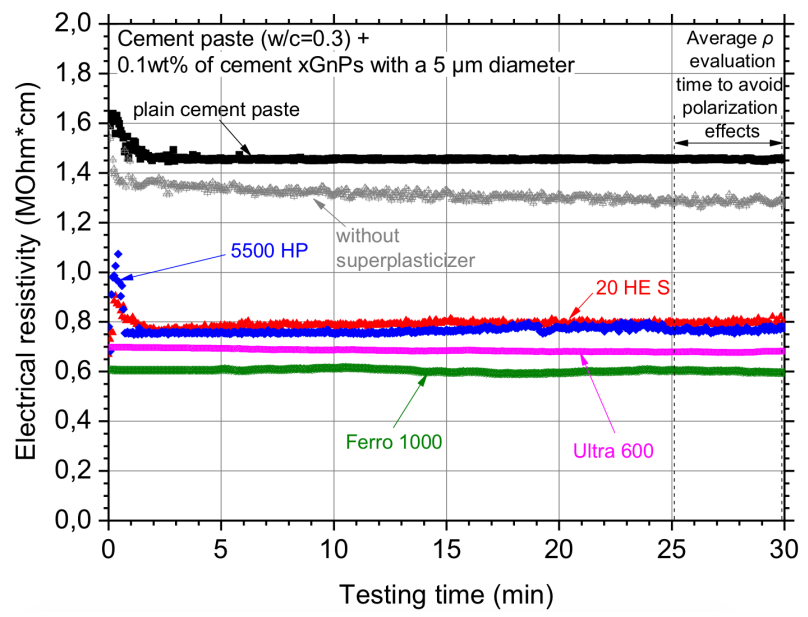

Fig 3. Typical electrical resistivity over testing time curves of the investigated nanocomposites with different superplasticizer types.

The average electrical resistivity values of the investigated samples along with their standard deviation are depicted in
Fig. 4. It is evident that the nanocomposites, regardless of the superplasticizer type employed for $\mathrm{xGnPs}$ dispersion, demonstrate lower electrical resistivity values compared to the reference material. This proves that the $\mathrm{xGnPs}$ can be used to improve the electrical properties of cement-based materials. The results of the electrical resistivity can be associated with the $\mathrm{xGnPs}$ dispersion state, i.e. the lower the electrical resistivity, the more uniform the $\mathrm{xGnPs}$ dispersion into the cementitious matrix. The cementitious materials exhibit insulator characteristics, therefore, the incorporation of a conductive reinforcement, such as the xGnPs, is expected to develop a conductive network inside the cementitious matrix providing the ability to pass electrical current through its body more effectively. The more uniform the $\mathrm{xGnPs}$ distribution is, the higher the electric paths that are developed inside the material are, which finally results to a global lower resistivity of the nanocomposite. As expected, the nanocomposites where dispersion was performed only with the application of ultrasonic energy (without the use of a dispersant agent) exhibit a slightly lower electrical resistivity compared to plain cement paste, demonstrating that the incorporation of a dispersing agent is necessary to homogeneously distribute the xGnPs. The $\mathrm{xGnP} /$ nanocomposites with the $20 \mathrm{HE} \mathrm{S}$ dispersant, based on polycarboxylate ester, exhibit the highest electrical resistivity among the nanocomposites with the different superplasticizer types. Furthermore, the results of those samples have a rather large scatter which is a clear indication of their non-uniformity due to the agglomeration of the xGnPs. The nanocomposites dispersed using the polycarboxylate polymer superplasticizers (Viscocrete ${ }^{\circledR}$ $5500 \mathrm{HP}$, Ultra 600 and Ferro 1000), exhibit the lowest resistivity, demonstrating that they can be employed to disperse the $\mathrm{xGnPs}$. Comparing the polycarboxylate polymer based dispersants, the Viscocrete ${ }^{\circledR} 5500$ HP depict a remarkable decrease in resistivity of approximately $48 \%$ against the reference samples. However, the standard deviation is quite large showing that there is probably an inhomogeneity, possibly caused by the presence of some xGnPs bundles. On the contrary, Ultra 600 presents the lowest standard deviation with a similar electrical resistivity decrease $(\sim 46 \%)$. The lowest $\rho$, around $52 \%$ from the initial value of the plain cement paste, and one of the lowest standard deviation values were achieved using Ferro 1000 as the dispersing agent. The experimental results in the present study show that a polycarboxylate polymer based superplasticizer is necessary for the effective dispersion of the $\mathrm{xGnPs}$ for cement-based materials.

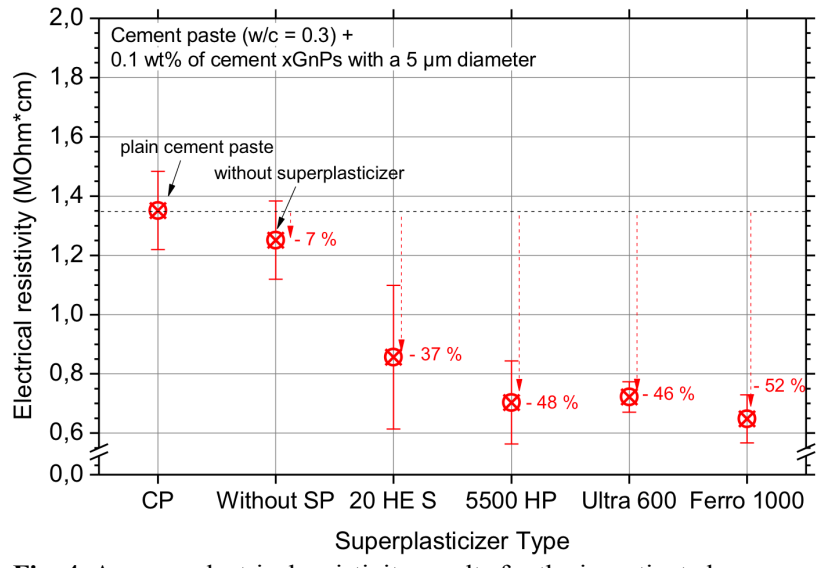

Fig. 4. Average electrical resistivity results for the investigated nanocomposites. 


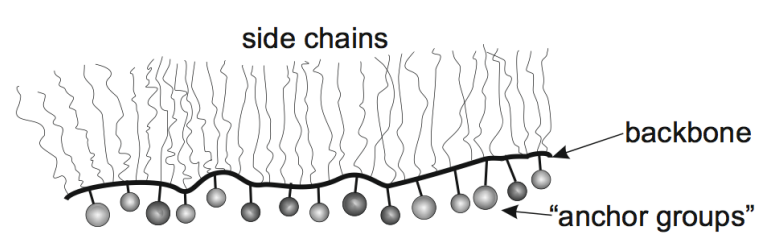

Fig. 5. Schematic representation of the typical molecular structure of polycarboxylate based superplasticizers.

The polycarboxylate based superplasticizers are macromolecular surfactants and their molecules look like a comb [18]. Their structure typically consists of an adsorbing anionic backbone and non-ionic side chains that aren't adsorbed [19], Fig. 5. According to Guardia et al. [20] nonionic surfactants can be used to successfully disperse graphene in water at significant concentrations. Additionally, anionic surfactants have been found effective in dispersing and stabilizing graphene particles in aqueous solutions even at low concentrations [21]. Possibly the combined function of both the anionic and the non-ionic components of the polycarboxylate based superplasticizers is what makes them effective as dispersing agents for graphene nanoplatelets in an aqueous solution. The superplasticizer molecular structure has several components and can be easily modified. Typical variations in their structure is the length of the backbone and side chains, the number of side chains and the nature of the monomers making up either the backbone or the side chains [22]. They are known to absorb on the cement grains and induce steric hindrance between surfaces which reduces or suppresses the otherwise attractive interparticle van der Waals forces [18]. Similar attractive van der Waals forces are developed between the graphene nanoplatelets that lead to their agglomeration. It should be noted that for a steric dispersant to be efficient, it must first be adsorbed [22]. The anchoring groups, which can be tightly adsorbed on the surface, are composed by high polarity groups such as $-\mathrm{COOH},-\mathrm{COO}^{-},-\mathrm{OH}$, etc. They develop a strong interaction with the cement particles due to hydrogen bond, covalent bond and van der Waals forces [23]. On the other hand, the side chains are responsible for the dispersing function of the polycarboxylate based superplasticizers as they are forming a layer on the outside surface, acting as a protection barrier [23]. They facilitate with the dispersion of the nanoparticles by forming a protective layer and preventing them from being coagulated and form agglomerates. It was found that typically the side chains are sticking out into the solution in a rather coiled conformation, with a characteristic thickness varying between 1.5 and $5 \mathrm{~nm}$ [24]. In principle, superplasticizers with longer side chains exhibit highest dispersing capability [25]. This is possibly because they impose larger distances between the particles, thus weakening the interparticle forces. Among the different polycarboxylate polymer based superplasticizers that were investigated in this study the ViscoCrete ${ }^{\circledR}$ Ferro-1000 is considered to be the most powerful. It has the capability to provide improved workability for a long time and strong water reduction. The length of the polymer side chains possibly plays an important role on its dispersing capability.

\section{Conclusions}

The dispersing efficiency of four different polycarboxylate based superplasticizers, based on either polycarboxylate ester or polycarboxylate polymers, for the disentanglement of exfoliated graphene nanoplatelets (xGnPs) used as reinforcement in cement based materials was investigated. The use of a dispersing agent (superplasticizer) was found to be an essential component for the uniform dispersion of the xGnPs. The employed polycarboxylate ester superplasticizer was found to be less suitable for the $\mathrm{xGnPs}$ dispersion, when compared to the investigated polycarboxylate polymer based dispersants. Among the different polycarboxylate polymer based superplasticizers studied, the ViscoCrete ${ }^{\circledR}$ Ferro-1000 was found to be the most effective to uniformly distribute the xGnPs. This is possibly due to the presence of longer side chains which is known to improve the dispersing efficiency of these type of superplasticizers. The combined function of both the anionic and the non-ionic components of the polycarboxylated based superplasticizers is possibly what makes them effective as dispersing agents for graphene nanoplatelets in an aqueous solution.

\section{Acknowledgments}

The author would like to acknowledge the financial support of IKY fellowships of excellence for postgraduate studies in Greece - Siemens Program.

\section{References}

1. C. Lee, X. Wei, J. Kysar and J. Hone, Science, 321, 385 (2008).

2. X. Du, I. Skachko, A. Barker and E.Y. Andrei, Nat. nanotechnol., 3, 491 (2008).

3. M. Pumera, Chem. Soc. Rev., 39, 4146 (2010).

4. Z. Yang, R. Gao, N. Hu, J. Chai, Y. Cheng, L. Zhang, H. Wei, E. S.W. Kong, Y. Zhang, Nano-Micro Lett., 3, 272 (2011).

5. S.G. Prolongo, A. Jiménez-Suárez, R. Moriche and A. Ureña, Eur. Polym. J., 53, 292 (2014).

6. A. Yazdanbakhsh, Z.C. Grasley, B. Tyson and R.K. Abu Al-Rub, ACI Special Publication, 267, 21 (2009).

7. A. Yazdanbakhsh, Z.C. Grasley, B. Tyson and R.K. Abu Al-Rub, Transp. Res. Record, 2142, 89 (2010).

8. K. Wille and K.J. Loh, Nanoengineering Transp. Res. Record, 2142, 119 (2010).

9. F. Collins, J. Lambert and W.H. Duan, Cement Concrete Comp., 34, 201 (2012).

10. O. Mendoza, G. Sierra and J.I. Tobón, Constr. Build. Mater., 54, 550 (2014).
11. T. Manzur and N. Yazdani, KSCE J. Civ. Eng., 19, (2015).

12. N. Zohhadi, N. Aich, I.A. Khan, F. Matta, N.B. Saleh and P. Ziehl, Proc. 4th Int. Symp. on Nanotechnol. in Constr. NICOM 4, Agios Nikolaos, Greece, (2012).

13. H. Alkhateb, A. Al-Ostaz, A. Cheng and X. Li. J. Nanomech. Micromech., 3, 67 (2013).

14. V.A. Coleman, R. Knut, O. Karis, H. Grennberg, U. Jansson, R. Quinlan, B.C. Holloway, B. Sanyal and O. Eriksson, J. Phys. D Appl. Phys., 41, 062001 (2008).

15. J.-L. Le, H. Du and S.D. Pang, Compos. Part B-Eng., 67, 555 (2014).

16. H. Du and S.D. Pang, Cement Concrete Res., 76, 10 (2015).

17. S. Wen and D.D.L. Chung, Cement Concrete Res., 31, 291 (2001).

18. H. Wu, H. Guo, J. Lei, R. Zhang and Y. Liu, Front. Chem. China, 2, 322 (2007).

19. R.J. Flatt, I. Schober, E. Raphael, C. Plassard and E. Lesniewska, Langmuir, 25, 845 (2009). 
20. L. Guardia, M.J. Fernandez-Merino, J.I. Paredes, P. SolisFernandez, S. Villar-Rodil, A. Martinez-Alonso and J.M.D. Tascon, Carbon, 49, 1653 (2011).

21. L. Liu, B. Gao, L. Wu, Y. Sun and Z. Zhou, Chem. Eng. J., 262, 1187 (2015).

22. C. Giraudeau, J.-B. d'Espinose de Lacaillerie, Z. Souguir, A. Nonat and R.J. Flatt, J. Am. Ceram. Soc., 92, 2471 (2009).

23. C. Miao, Q. Tian, Q. Ran and J. Liu, Proc. 1st Int. Con. on Microstr. Related Durability of Cem. Comp., Nanjing, China, (2008).

24. A. Kauppi, K.M. Andersson and L. Bergström, Cement Concrete Res., 35, 133 (2005).

25. K. Yamada, T. Takahashi, S. Hanehara and M. Matsuhisa, Cement Concrete Res., 30, 197 (2000). 